\title{
THE CHARGE TRANSPORT IN AN ELECTROSTATIC BELT GENERATOR
}

\author{
A. VERMEER and B. A. STRASTERS \\ Fysisch Laboratorium, Rijksuniversiteit, Utrecht, The Netherlands
}

Received 21 October 1975

\begin{abstract}
The fluctuations in the charge transport system of an EN Tandem Van de Graaff accelerator have been investigated by means of a frequency spectrum analyser. Frequency spectra of the terminal ripple, the short-circuit current and the voltage at the belt charge screen have been measured. Also the correlation
\end{abstract}

\section{Introduction}

As part of a study to reduce the fluctuations in the terminal voltage of a Van de Graaff generator an investigation was made into the charge transport by the charging belt. The data obtained can be used in the design and development of a voltage stabilization system. In a Van de Graaff generator charge is transported to the high voltage terminal by means of a moving belt (or a pellet chain), and this moving charge constitutes a current which charges the terminal capacitor. A Van de Graaff generator can therefore be described as an almost ideal dc current source, due to the high insulation value of the belt. Irregularities in the charge transport, e.g. by a varying speed of the belt, a non-uniform charge distribution on the belt, corona discharges along the belt, etc., manifest themselves as voltage fluctuations on the terminal voltage. To obtain an insight into the charge transport the fluctuations in the short-circuit current, in the belt charge voltage, and in the terminal voltage were examined. To determine the frequency spectrum of the fluctuations use was made of a frequency analyser. These measurements show that the frequency spectrum consists of a. fundamental frequency (determined by the revolution frequency of the belt) and harmonics. Harmonic components up to the twentieth are observed; usually, however, only the first four harmonics are relatively strong. Because of the smoothing action of the terminal capacitor the ripple in the terminal voltage is determined mainly by the lower harmonic components. Firequencies were found which were non-harmonic to the revolution frequency of the belt. In this article the contribution of the various mechanisms to the frequency spectrum will be examined. Therefore the lateral vibrations of the belt and the characteristic frequencies of the belt system were considered. It appears that a moving belt can show, as in the case of a string, a function is examined between the last two signals. The mechanisms which can cause the measured fluctuations are discussed. The influence of fluctuations in the belt velocity and of vibrations is apparent.

frequency spectrum with many harmonics. If a source is present which has a frequency in the neighbourhood of one of the belt vibration frequencies, belt vibrations will be excited. As will be seen in the following, the rotation of the belt can be such a source of vibrations. The belt vibrations cause fluctuations in the belt velocity, giving rise to variations in the charge transport. A formula is derived for the influence of these velocity variations on the current delivered to the terminal capacitor. Although the presented measurements have been performed on an EN Tandem Van de Graaff generator, with a tank gas mixture of $80 \% \mathrm{~N}_{2}$ and $20 \% \mathrm{CO}_{2}$ at $15 \mathrm{~atm}$, one can expect the results to have a wider validity.

\section{The belt charge transport}

In a Van de Graaff generator the charge, put into the belt by a corona discharge, is collected at the high voltage terminal by means of the collector screen. Because of irregularities in the transport the terminal voltage shows a ripple. By means of an amplifier

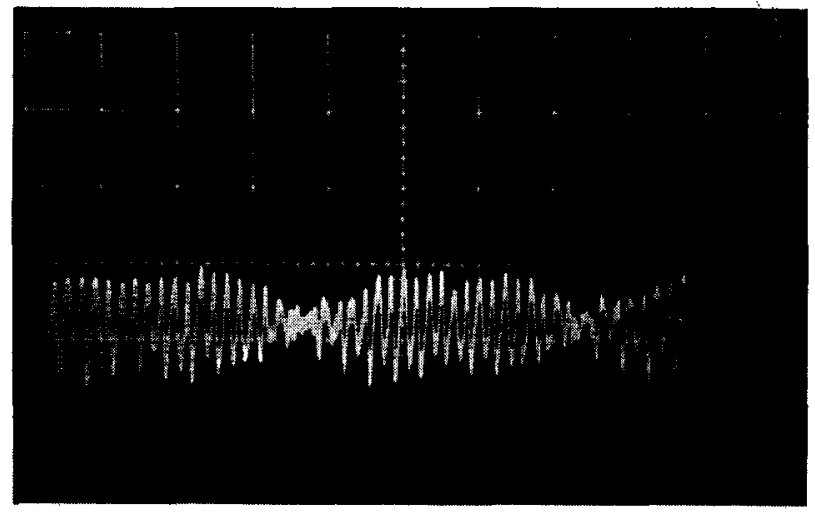

Fig. 1. The repetitive terminal ripple pattern. Vertical: $4 \mathrm{kV} / \mathrm{div}$. $i_{\text {upch }}=100 \mu \mathrm{A}$. Horizontal: $1 \mathrm{~s} /$ div. 
connected to the pick-up plate the ripple voltage is measured. The frequency response of the measurement system is flat from 0.1 to $200 \mathrm{~Hz}$. The measured ripple voltage shows a repetitive pattern as is seen in fig. 1. The frequency spectrum of this pattern, which extends from about 2.5 to $50 \mathrm{~Hz}$ is not suitable for accurate analysis by using standard filtering techniques. Therefore another technique was tried, using a special tape recorder with a closed loop tape (the length of the tape is $90 \mathrm{~cm}$ ). During the recording the tape makes one full revolution at a speed of $0.38 \mathrm{~mm} / \mathrm{s}$. To analyse the recorded spectrum the tape loop is played back at a speed 250 times as fast. This transforms the real frequency spectrum, which extends to about $50 \mathrm{~Hz}$, into a frequency range which is 250 times higher. This frequency-transformed spectrum can be accurately analysed with a spectrum analyser. In this case a Tektronix plug-in unit spectrum analyser type 1L5 was used. The analysing equipment, consisting of the tape recorder and the frequency analyser, was calibrated with the aid of a signal generator. By dividing the frequency scale of the transformed spectrum by 250 , the real frequency spectrum is obtained. Fig. 2a shows schematically the belt system and the points $\mathrm{A}, \mathrm{B}$ and $\mathrm{C}$ with which the analysing equipment (see fig. $2 \mathrm{~b}$ ) can be connected. Because of the interest in the charge transport system, the fluctuations at the belt charge screen were measured (point $\mathrm{A}$; with a Tektronix probe $\mathrm{P}$ 6013A) and the fluctuations in the short-circuit current (point $B$; shorting rod moved in) at the terminal collector screen. At point $\mathrm{C}$ the terminal ripple can be measured. The measurements (of which the analysis is rather time consuming) were performed at upcharge currents of 50 , 100 and $200 \mu \mathrm{A}$. In figs. 3, 4 and 5 the results are shown of a measurement with an upcharge current of $50 \mu \mathrm{A}$. In all three cases we see a fundamental frequency with harmonics. The fundamental frequency of $2.6 \mathrm{~Hz}$ corresponds to the belt revolution period. Nonharmonic frequency components are also observed. The thin lines in the figures are drawn as a guide for the eye. This general picture also applies for other values of the upcharge current. Some measurements were also carried out with zero upcharge current and a similar frequency spectrum was observed, due to the selfcharging of the belt. Inspection of the frequency spectrum indicates that it can be divided into three parts. The low-frequency part extends to about $10 \mathrm{~Hz}$, including the fourth harmonic of the belt frequency. The high-frequency part consists of components around the 15th harmonic, and in between these and the lowfrequency part there are components of which the amplitudes are weaker and mostly of the same order of
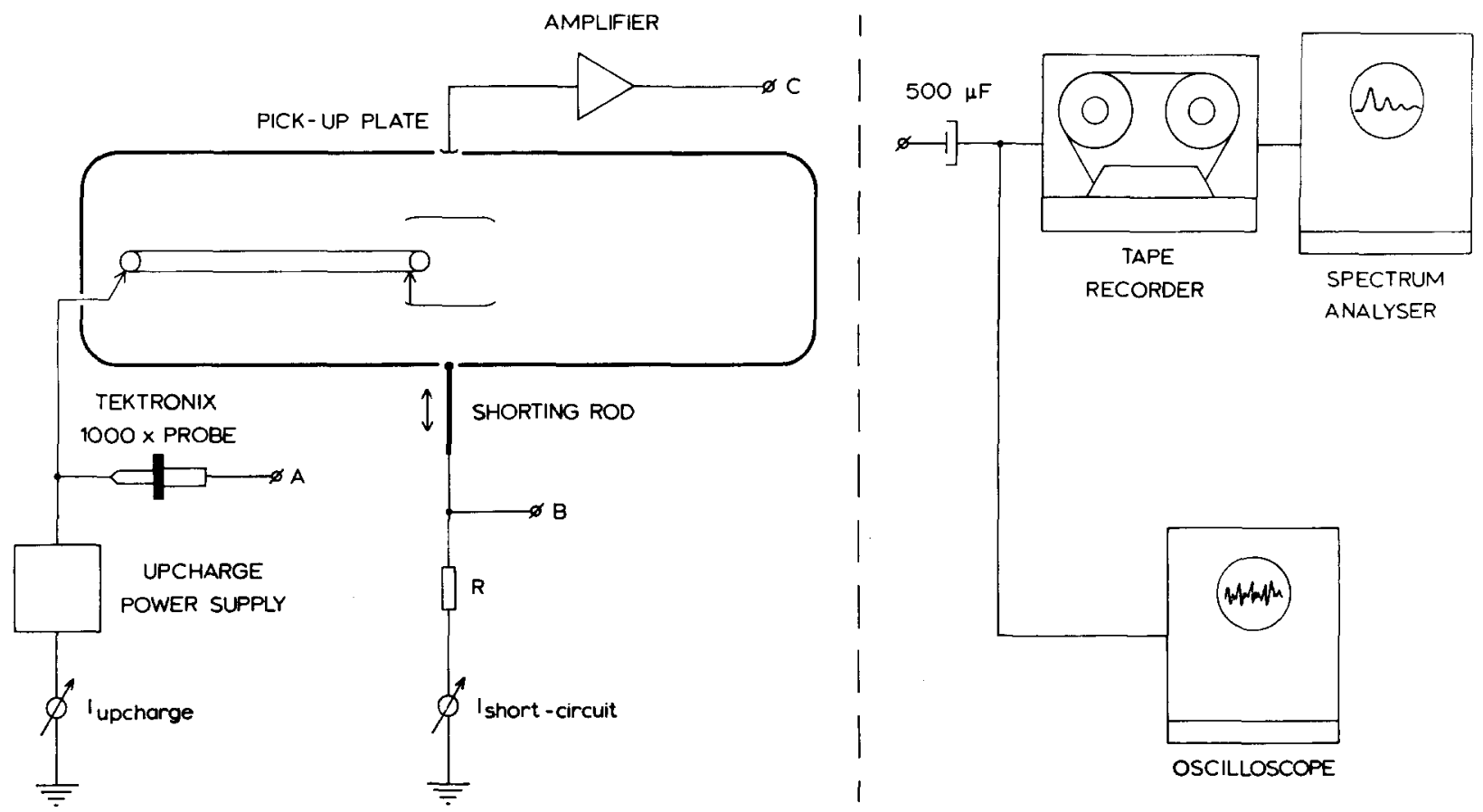

Fig. 2. (left) The belt system with the measuring points $A, B$ and $C$. The resistor $R$ can have different values depending of the sensitivity of the analysing equipment. Common values are $R=220,500$ or $3300 \Omega$ (right) The analysing equipment. 
magnitude. The frequency spectrum of the belt charge screen voltage $V_{\text {upch }}$ has the same structure as at the terminal collector screen current $i_{\mathrm{sc}}$. This is clearly demonstrated in fig. 6 , where on the same graph is plotted the spectrum of the belt charge sreen voltage and the short-circuit current. To show the coherence between the mentioned signals the cross-correlation function was determined with the aid of a PAR 101A correlation function computer. In fig. 7 the crosscorrelation function is shown. The regularly fluctuating curve indicates the existing correlation between both signals. The interpretation of the curve is discussed in sect. 3.3.

It should be remembered that in the frequency spec-

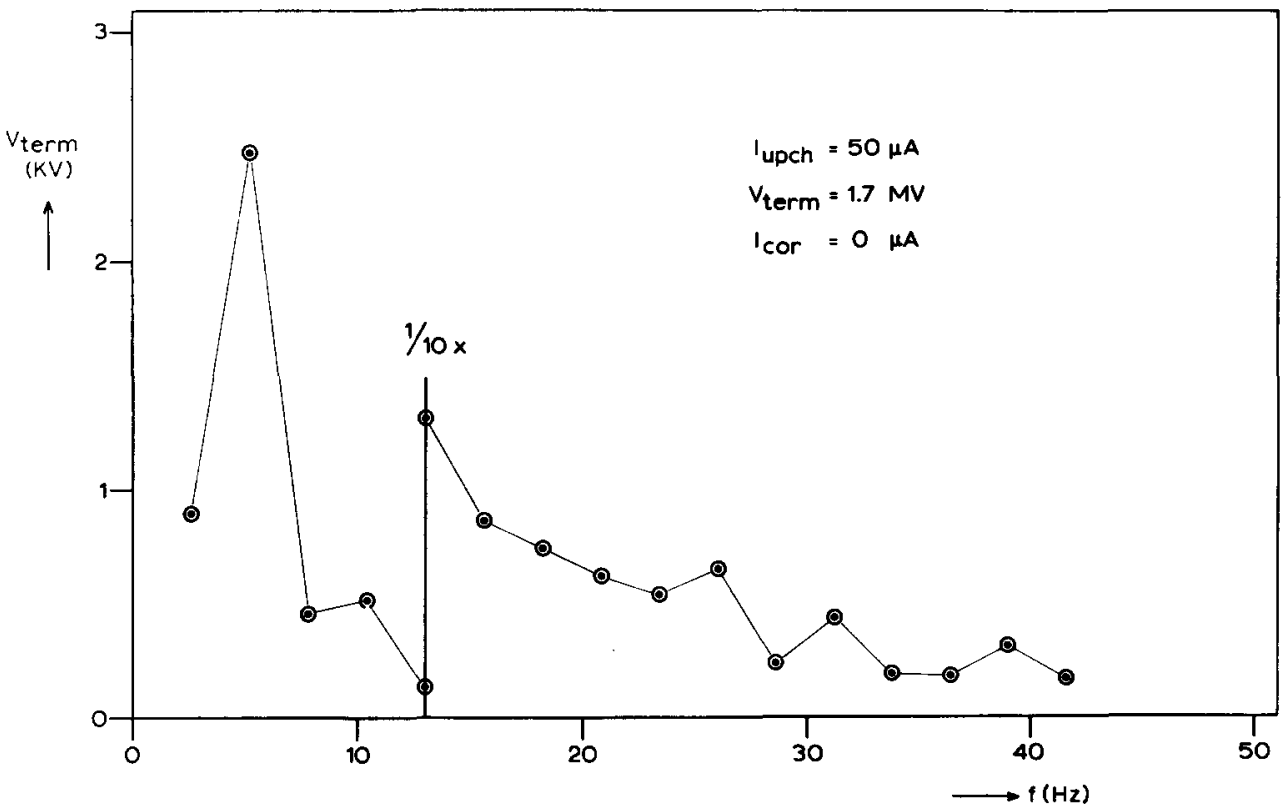

Fig. 3. The frequency spectrum of the terminal ripple for an upcharge current of $50 \mu \mathrm{A}$.

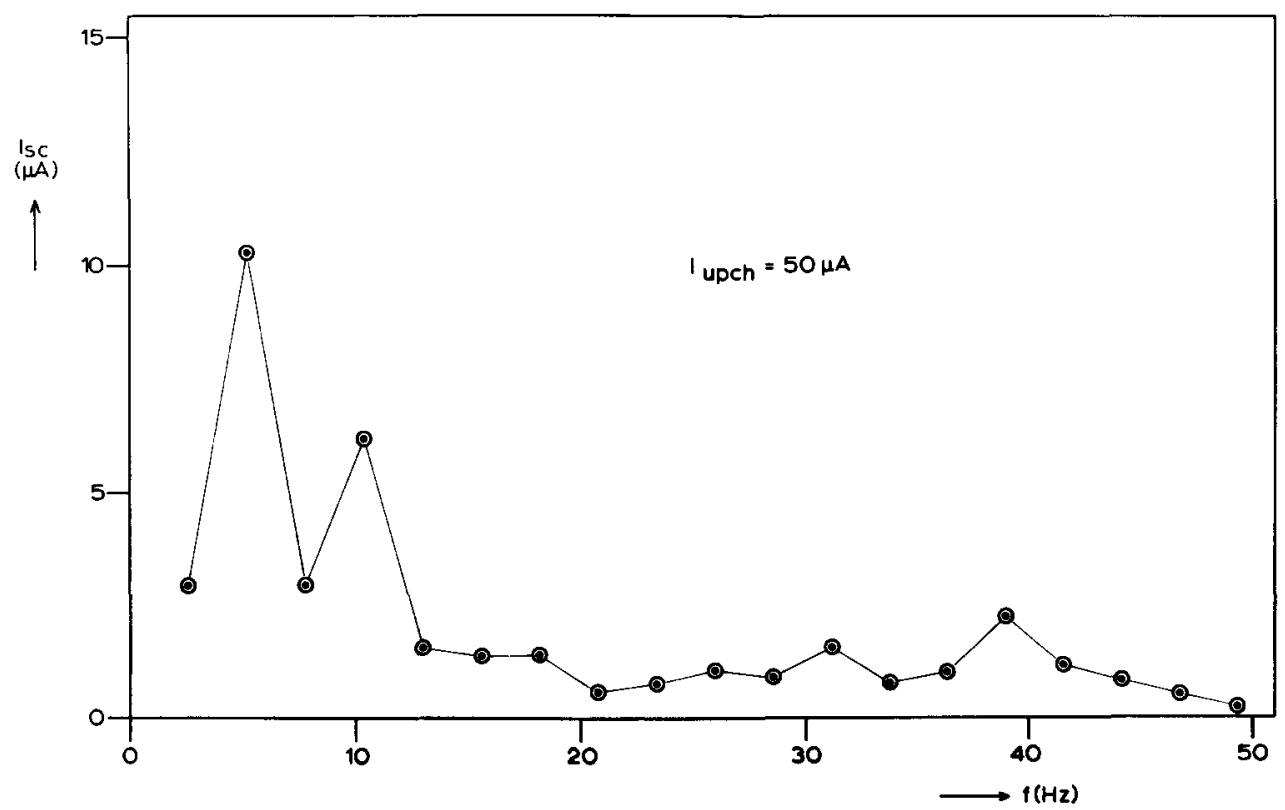

Fig. 4. The frequency spectrum of the short-circuit current measured over the resistor $\mathrm{R}$. The upcharge current was $50 \mu \mathrm{A}$. 
trum of the short-circuit current the ratio of the amplitudes is correct. The higher-frequency components of the measured terminal voltage fluctuations, however, are relatively attenuated, due to the terminal capacitance $C_{\mathrm{T}}$ (about $150 \mathrm{pF}$ ). It is possible to calculate the spectrum of the terminal ripple from the frequency spectrum of the short-circuit current by multiplying the amplitude of every component by $1 / \omega C_{\mathrm{T}}$. The result in fig. 8 shows the good agreement between the measured and calculated spectrum. We were also interested in the influence of the belt charge current on the terminal ripple. The results of these measurements are collected in fig. 9. For the largest part of the spectrum, the maximum difference in

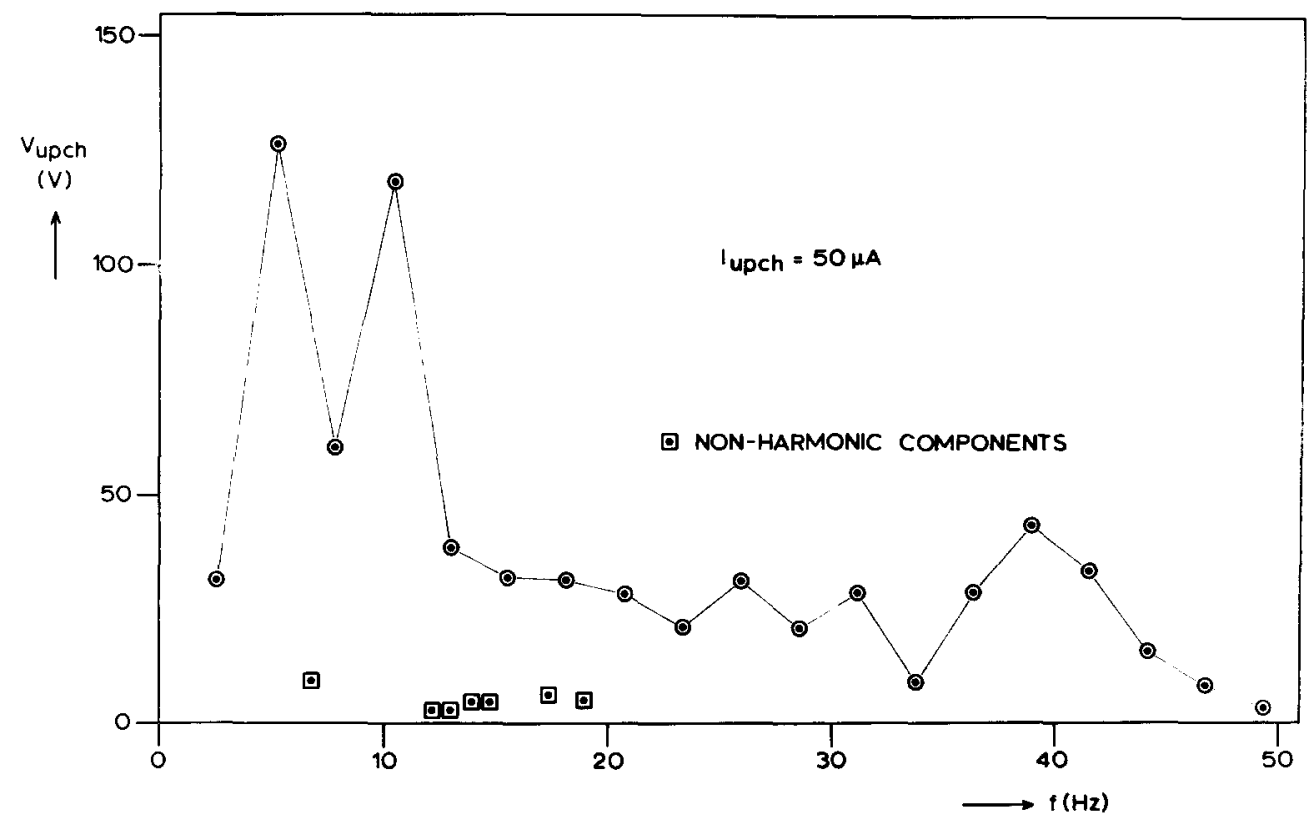

Fig. 5. The frequency spectrum of the voltage at the belt charge screen at an upcharge current of $50 \mu \mathrm{A}$.

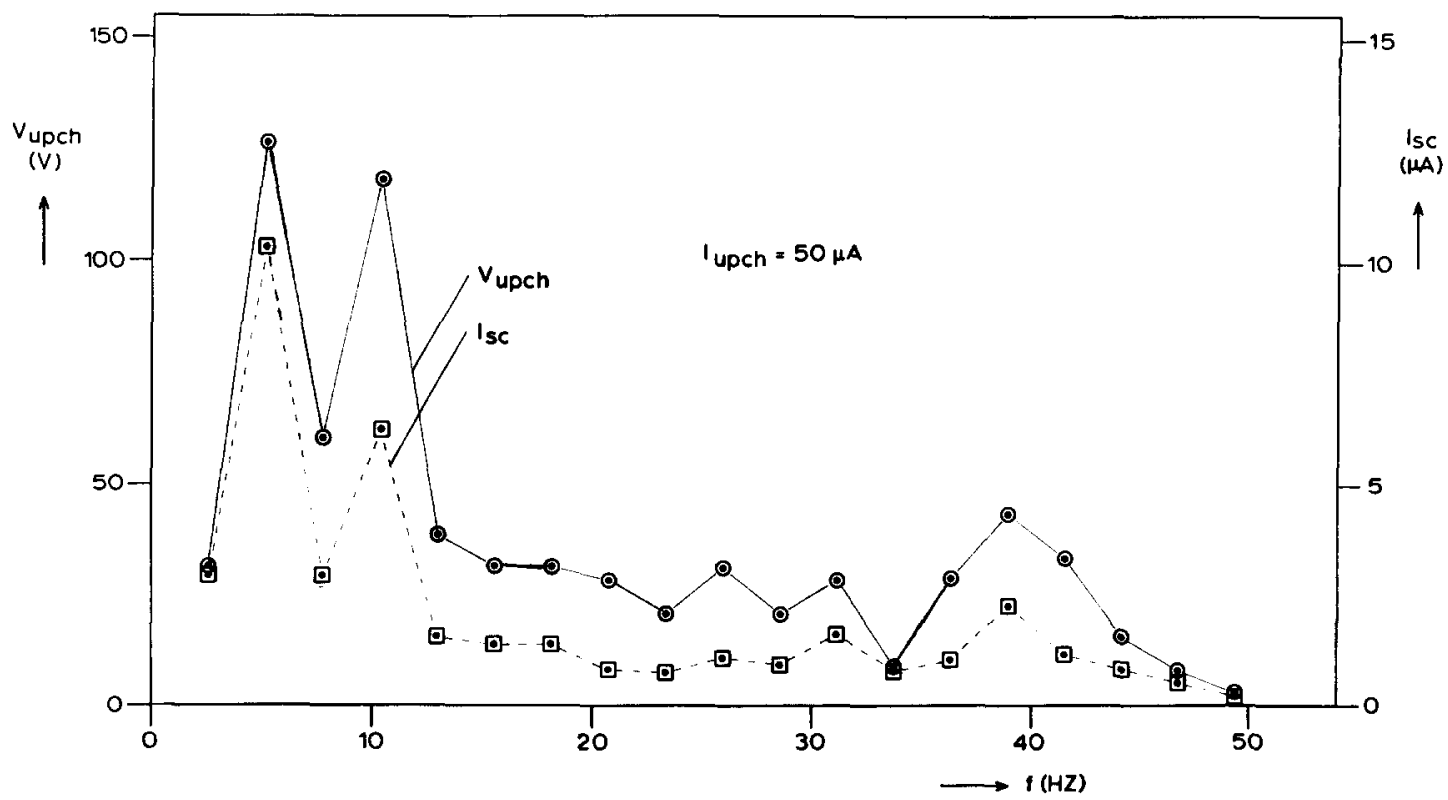

Fig. 6. The frequency spectrum of the belt charge screen voltage and the short-circuit current plotted in one graph; $i_{\text {upeh }}=50 \mu \mathrm{A}$. 
amplitude for corresponding harmonics is not more than $50 \%$. The amplitudes of the lower-frequency components in the case of $200 \mu \mathrm{A}$ upcharge current are clearly lower than at $50 \mu \mathrm{A}$ and $100 \mu \mathrm{A}$.

\section{Discussion}

As can be seen from the spectra there always appears a fundamental frequency (the so-called belt frequency) of $2.6 \mathrm{~Hz}$. One would expect that an ideal endless belt would possess no "marks" to indicate when one revolution is finished. However, because of inhomogeneities or imperfections of the belt material, the belt has a structural pattern, which repeats periodically during each revolution. Under certain conditions vibrational modes of the belt system can be excited in resonance with the harmonics of the belt frequency. The clearly audible periodic changes in the belt sound of the EN Tandem have the same period as the belt period measured from the spectra. This suggests that during one revolution of the belt there are "heavy points" in the running belt.

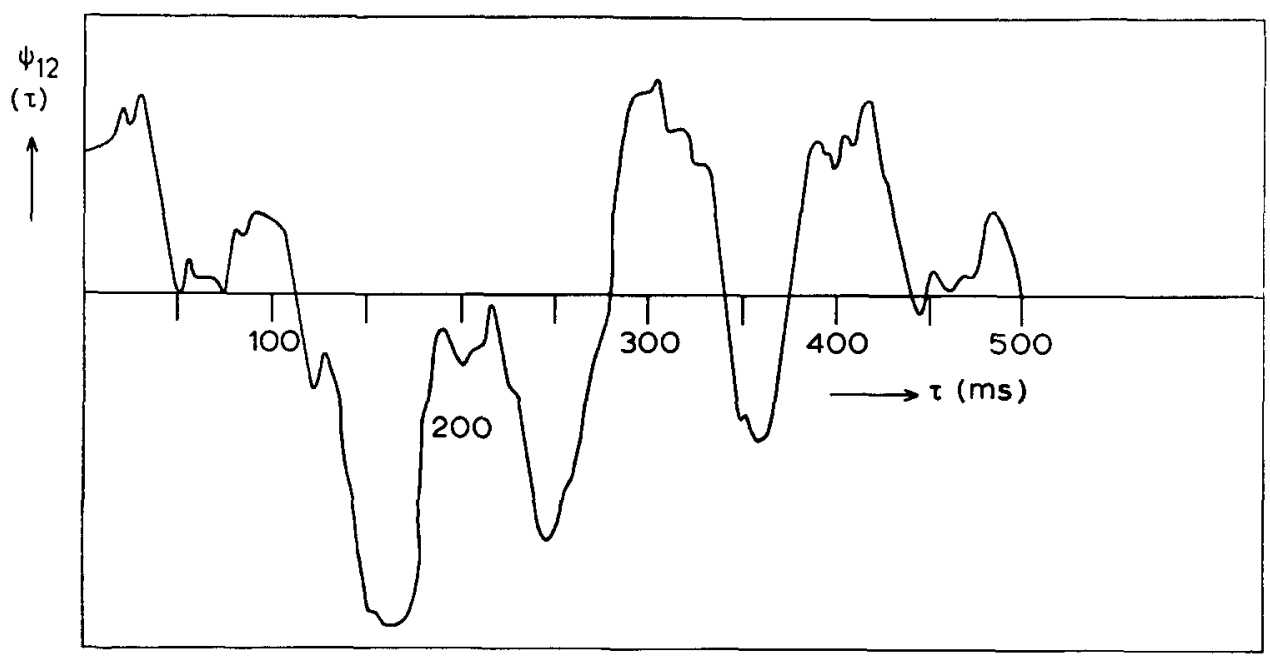

Fig. 7. The cross-correlation function between the signals $V_{\mathrm{upch}}$ and $i_{\mathrm{sc}}$, for $i_{\mathrm{upch}}=100 \mu \mathrm{A}$.

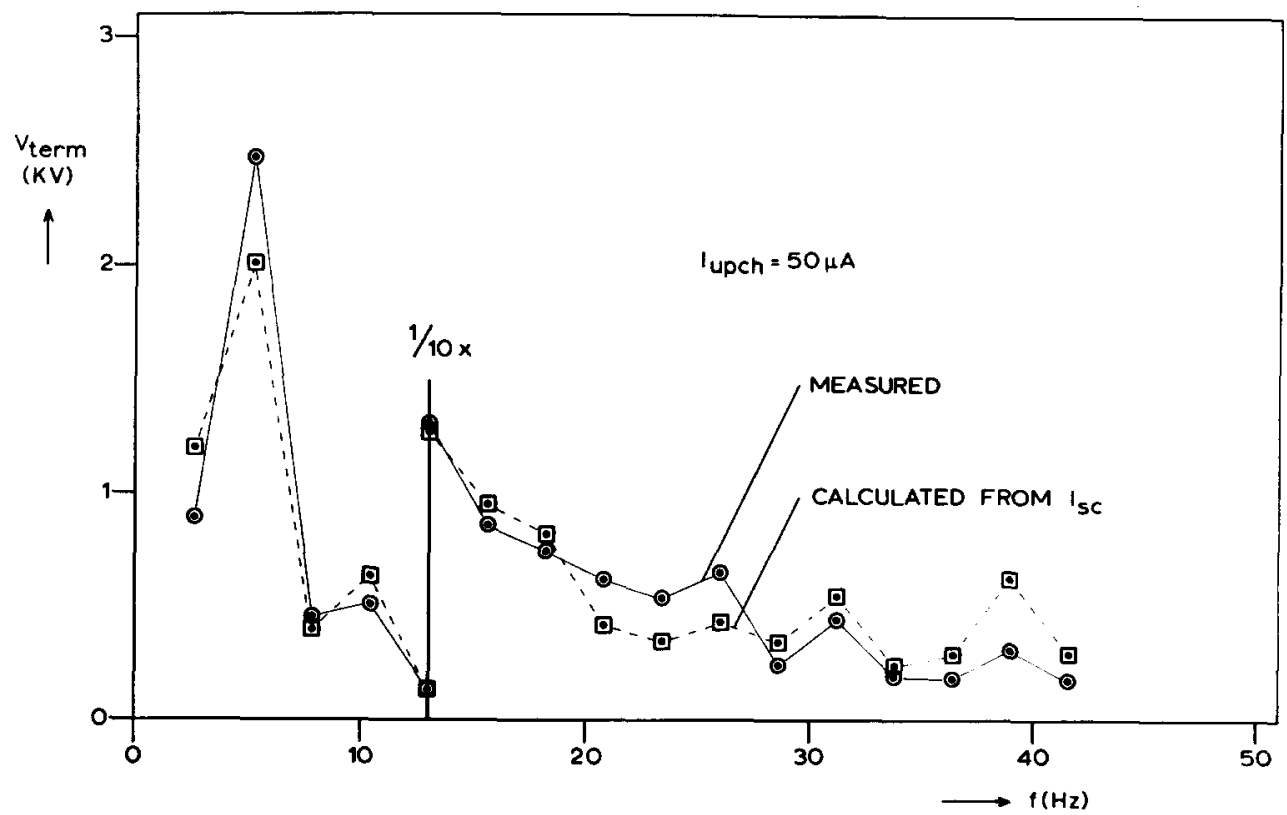

Fig. 8. The measured and calculated terminal ripple at an upcharge current of $50 \mu \mathrm{A}$. 


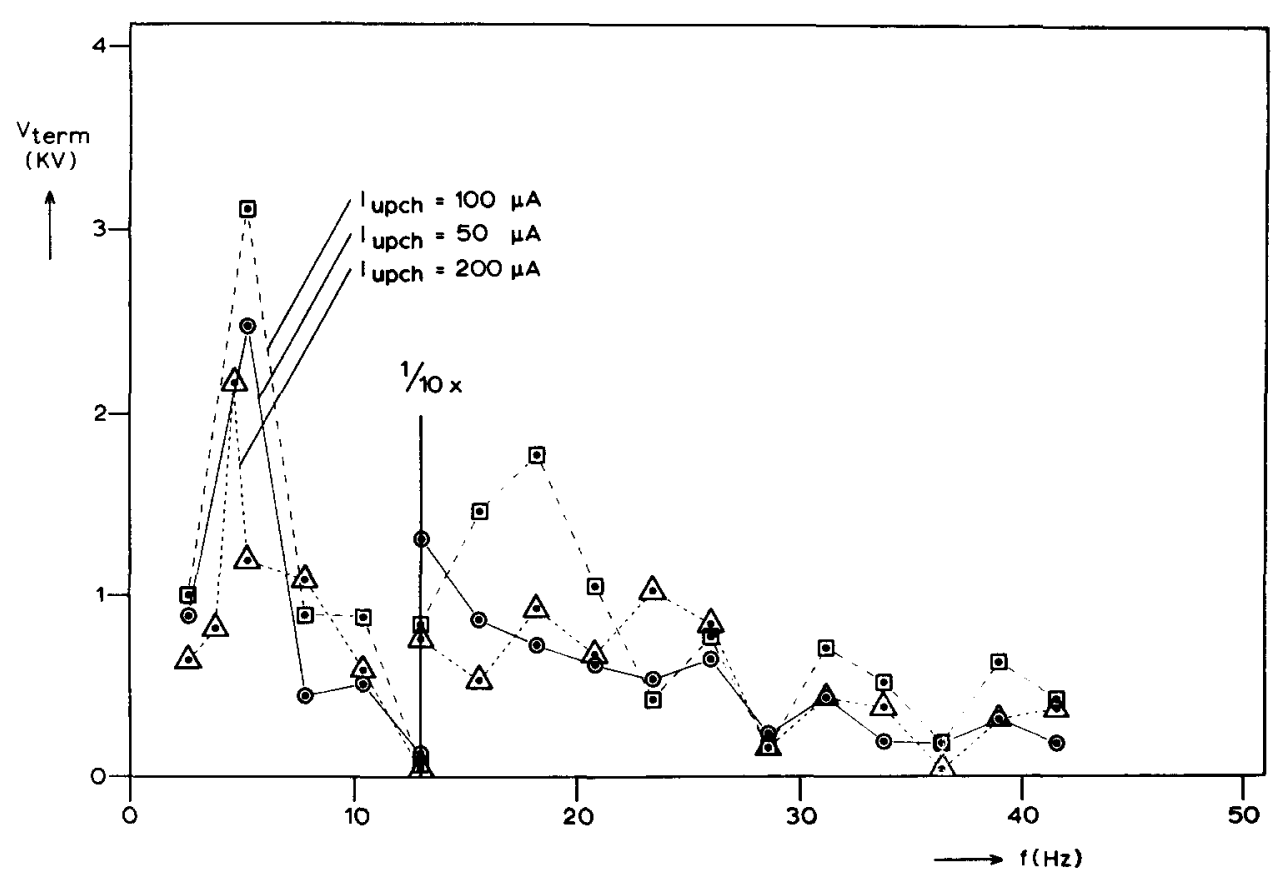

Fig. 9. The spectrum of the terminal ripple $V_{\text {term }}$ at different values of the upcharge current.

In order to investigate this we measured with an oscilloscope the current taken by the belt motor (3-phase, 16.6 hp, 2880 r.p.m.). Fig. 10 shows the fluctuations in the phase current measured with a current transformer. These fluctuations have a period equal to the belt period and the difference between the maximum and average phase current is about $3 \%$. For an asynchronous motor, such as the belt motor, this is also the magnitude of the variation in the revolution speed, so that the velocity of the charge transport is not precisely constant. We consider the consequences of this point in sections 3.2 and 3.3. The variations in the speed of the belt motor were also checked by means of a stroboscope; inspection of the rotating belt motor pulley revealed periodic variations in the motor speed having a frequency equal to the belt frequency.

\subsection{THE FREQUENCY SPECTRUM}

To explain the measured frequencies it is necessary to consider the characteristic frequencies of the belt system. First consider the frequencies of the vibrating belt given by eq. (4) in the appendix. These frequencies are:

$$
v_{n}=\frac{n}{2 l} \frac{c^{2}-v^{2}}{c}, \quad n=0,1,2,3, \text { etc } .
$$

with $c=\sqrt{ }(S / \rho)=55 \mathrm{~m} / \mathrm{s}$, as $S=$ the tension in the string, $\rho=$ the mass per unit length $(\rho=1.7 \mathrm{~kg} / \mathrm{m}$ in the present case) and $l=$ the belt length. The HVEC manual gives a belt tension of $5000 \mathrm{~N}$ at the upper and lower bearing for an EN belt, so the tension in each part of the belt is $5000 \mathrm{~N}$.

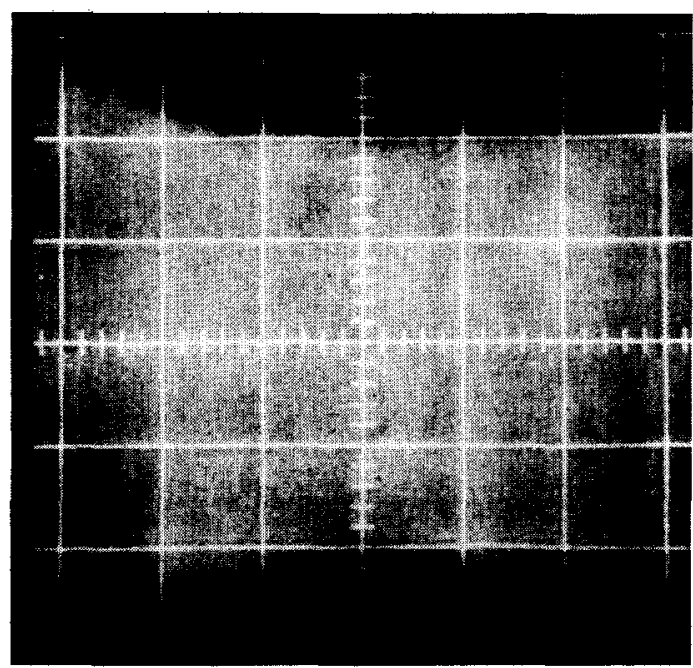

Fig. 10. Fluctuations in the current taken from the mains by the belt motor. The picture is taken with a storage scope. Vertical: arbitrary units; the distance between two consecutive subdivision lines is $\Delta i$ (belt motor). Horizontal: $250 \mathrm{~ms} / \mathrm{div}$. 
This yields, with a free length of the belt of $l=4.3 \mathrm{~m}$ and $v=23 \mathrm{~m} / \mathrm{s}$, the frequencies $v_{n}=5.3 n \mathrm{~Hz}(n=1,2$, $3, \ldots)$ or $v_{1}=5.3 \mathrm{~Hz}, v_{2}=10.6 \mathrm{~Hz}$, etc. Thus the belt can vibrate at these frequencies, giving rise to small corresponding fluctuations in the load of the belt motor. Unfortunately the frequencies $5.3 \mathrm{~Hz}$ and $10.6 \mathrm{~Hz}$ are nearly equal to the second and fourth harmonics of the belt frequency, respectively. This explains why the even harmonics, as the spectra show, are strongly excited. It would seem that the parameters of the belt have been unfortunately chosen. The velocity $v$ and the mass per unit length $\rho$ are constant for a given drive motor and belt, so the only parameter available for shifting the vibration frequencies is the tension $S$. However, the frequency varies only as the square root of $S$, and in addition the value of $S$ is also determined by mechanical considerations, as the tension must be high enough to give sufficient friction at the pulleys (the axes of which are vertical) to counteract the weight of the vertical belt. The maximum allowable strain sets an upper limit on the belt tension. Another limit is that the belt velocity $v$ must be smaller than the velocity $c$ of the waves along the belt. In the case of $v \geqslant c$ the belt cannot run in a stable condition, as is derived in refs. 1 and 2 . To be sure that no flapping of the belt will occur, it is safe to take the velocity $c$ much higher than the belt velocity $v$. In the present case at the mentioned belt tension $S$ one finds $c=55 \mathrm{~m} / \mathrm{s}$, so the condition for stable operation of the belt is amply fulfilled. A relatively strong component in all spectra is the 15 th harmonic, at about $40 \mathrm{~Hz}$. This is not the revolution frequency of the pulley (2850 r.p.m. corresponds to $47.5 \mathrm{~Hz}$ ) so that this component must have another origin, which can be found in the structure of the belt. In the belt an increase in the number of threads across the belt occurs at intervals of about $\left.60 \mathrm{~cm}^{3}\right)$. At a belt velocity of $23 \mathrm{~m} / \mathrm{s}$ this periodic increase of crossthread density represents an inhomogeneity which recurs with a frequency of about $40 \mathrm{~Hz}$. The other harmonics between the strong components in the beginning of the spectra and the 15 th harmonic can find their cause in inhomogeneities in the belt. After the belt has run for some time many "strips" across it become clearly visible, corresponding to irregularities in the belt. This "belt pattern" turns around with the belt frequency. Fourier analysis yields many components which are all harmonic to the belt frequency. Also, some spectra show some non-harmonic components. An explanation for these frequency components can be found in the fact that vibrations across the belt are also possible. A combination of the modes of vibration along and across the belt can give frequencies which are shifted with regard to the harmonic components.

\subsection{THE CHARGE TRANSPORT}

The charge sprayed onto the belt by the belt charge screen is transported by the belt to the terminal. It is of interest to examine what the influence is of variations in the belt velocity on the current $i_{\text {col }}$ at the terminal collector screen. Variations in this current give rise to fluctuations in the terminal voltage, the so-called terminal ripple. In fig. 11 a the principle of the charge transport by means of the belt is shown. In this case the charge is furnished to the belt at a time $t=t_{1}$ at a belt velocity $v_{1}$, while the charge is removed from the belt at a time $t=t_{2}$ at a velocity $v_{2}$, which differs from $v_{1}$. We will follow this process with the help of fig. 1 la. In the time interval $\Delta t_{1}$ between $t_{1}$ and $t_{1}+\Delta t_{1}$ the belt moves with a velocity $v_{1}$ over a distance $\Delta x_{1}$. The charge density furnished to the belt in that time interval is $\sigma_{1}=i_{\text {upch }} / b v_{1}$. The strip with charge density $\sigma_{1}$ arrives at the collector screen at the time $t_{2}=t_{1}+\tau_{1}$, with $\tau_{1}=l / v_{0}\left(v_{0}\right.$ is the average belt velocity and $l$ is the distance between upcharge and collector screen). The delivered current to the terminal is then $i_{\text {col }}=b \sigma_{1} v_{2}=i_{\text {upch }} v_{2} / v_{1}$. The fluctuating belt velocity is a periodic function of time and can be expanded in a

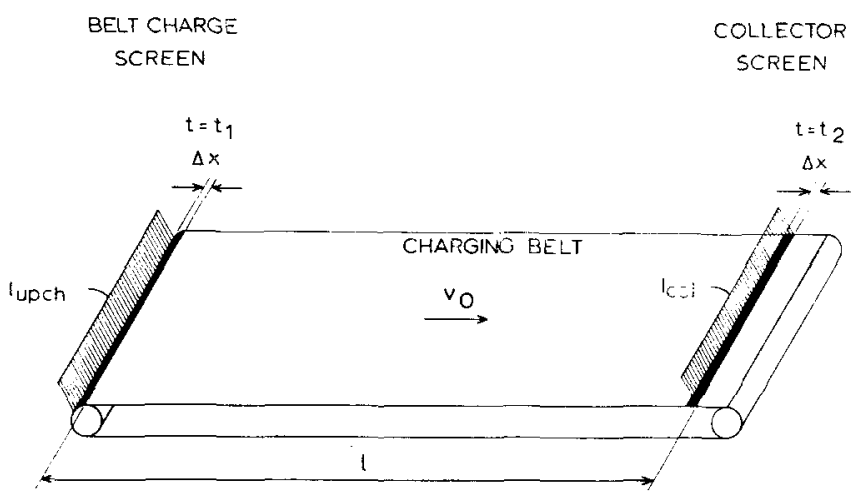

(a)

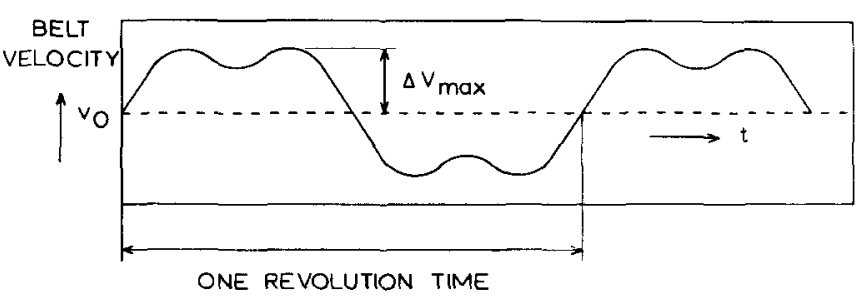

(b)

Fig. 11. (a) Schematic drawing of the belt generator. (b) Sketch of the belt velocity as a function of time. 
Fourier series. Because the function for the fluctuations in the belt velocity is an odd one (see figs. 10 and 11b), only sine terms can be taken into account, so $v=v_{0}+\sum_{n} \Delta v_{n} \sin n \omega t$, with $n=1,2,3 \ldots$ and $v_{n} / v_{0} \ll 1$. Substituting this velocity in the expression for $i_{\text {col }}$, we get:

$$
\frac{i_{\text {col }}}{i_{\text {upch }}}=\frac{1+\sum_{n}\left(\Delta v_{n} / v_{0}\right) \sin n \omega\left(t+\tau_{1}\right)}{1+\sum_{n}\left(\Delta v_{n} / v_{0}\right) \sin n \omega t} .
$$

Expanding the denominator into powers of the sine sum, and neglecting the higher order terms, yields the fluctuation $\Delta i_{\text {col }}$ in the collector current

$$
\begin{aligned}
\frac{\Delta i_{\text {col }}}{i_{\text {upch }}}= & \frac{2 \Delta v_{1}}{v_{0}} \cos \omega\left(t+\frac{1}{2} \tau_{1}\right) \sin \frac{1}{2} \omega \tau_{1}+ \\
& +\frac{2 \Delta v_{2}}{v_{0}} \cos 2 \omega\left(t+\frac{1}{2} \tau_{1}\right) \sin \omega \tau_{1}+ \\
& +\frac{2 \Delta v_{3}}{v_{0}} \cos 3 \omega\left(t+\frac{1}{2} \tau_{1}\right) \sin \frac{3}{2} \omega \tau_{1}+ \\
& +\frac{2 \Delta v_{4}}{v_{0}} \cos 4 \omega\left(t+\frac{1}{2} \tau_{1}\right) \sin 2 \omega \tau_{1}+\ldots
\end{aligned}
$$

Because the time $\tau_{1}$ is almost half of the belt period, all the even harmonics are nearly zero, because of the term $\sin n \omega \tau_{1}$, and only the odd harmonics remain. So the fluctuations in the belt velocity cannot be the cause of the strong second and fourth harmonics found in the spectra. For that another mechanism must be present, which we discuss in the next section. One can simply derive the ratio of the peak-to-peak value of the fluctuations in the collector current to the upcharge current, by summing up all contributions of the odd harmonics $\sum_{n} 2 \Delta v_{n}=\Delta v_{\max }, n=1,3,5, \ldots$, (see fig. $11 \mathrm{~b})$. We then find

$$
\left(\Delta i_{\mathrm{col}}\right)_{\mathrm{p}-\mathrm{p}} / i_{\mathrm{upch}}=4 \Delta v_{\max } / v_{0} .
$$

Since for an asynchronous motor the motor current is proportional to the angular velocity (over a small range), the belt velocity variations in percent are equal to the belt motor current variations. As already mentioned the variation in the belt motor current is about $3 \%$. From eq. (1) it follows that $\left(\Delta i_{\text {col }}\right)_{\mathrm{p}-\mathrm{p}} / i_{\text {upch }}$ is about $12 \%$. The peak-to-peak value of the fluctuation in the collector current, measured from the scope pictures, can differ considerably from this $12 \%$, because only the contribution of the odd harmonics is taken into account. But the selfcharging of the belt and
TABLE 1

Measured and calculated values for $\left(\Delta i_{\mathrm{col}}\right)_{\mathrm{p}-\mathrm{p}}$ at different upcharge currents.

$\begin{array}{ccc}i_{\text {upch }} & \left(\Delta i_{\text {col })_{\mathrm{p}-\mathrm{p}}}\right. & \text { Calculated value } \\ (\mu \mathrm{A}) & (\mu \mathrm{A}) & \text { from eq. }(1) \\ & & (\mu \mathrm{A})\end{array}$

$\begin{array}{rrr}50 & 26 & 6 \\ 100 & 30 & 12 \\ 200 & 27 & 24\end{array}$

the parasitic discharges along the belt also have their influence on the ripple in the collector current. Parasitic discharges, especially at higher loading of the belt, can have a smoothing effect on the collector current fluctuations. In table 1 measured and calculated values for $\left(\Delta i_{\mathrm{col}}\right)_{\mathrm{p}-\mathrm{p}}$ are collected.

\subsection{THE UPCHARGE AND COLLECTOR SCREEN CURRENT}

The belt transports charge which is sprayed onto the belt by means of the belt charge screen. It is therefore of importance to look into the influence of this charging proces on the terminal ripple. The principle of the charging process is schematically illustrated in fig. 12 . The upcharge power supply delivers charge which is sprayed onto the belt as a corona current. We can assume that the corona current $i_{\text {upch }}$ is constant. This is a realistic assumption because the current fluctuations from the HVEC current stabilized upcharge power supply have a magnitude of no more than $0.2 \%$. The

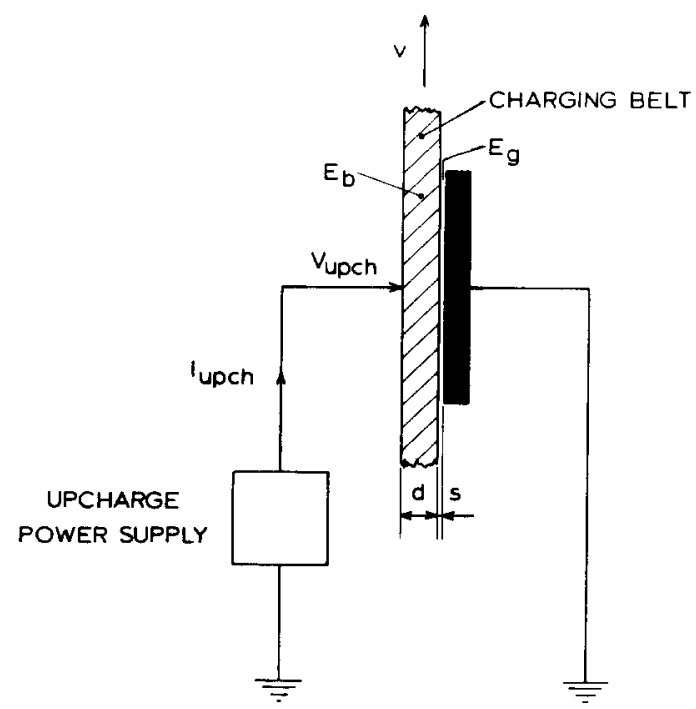

Fig. 12. The principle of the charging of the belt. 
charge density $\sigma$ on the belt is then $\sigma=i_{\text {upch }} / b v$. Because the belt velocity has small fluctuations, the charge density $\sigma$ also has a "ripple". The charge collected at the position of the belt charge screen in the time interval $\Delta t$ arrives at the collector screen in the terminal at a later time at different speed, and gives rise to the terminal ripple. Examination of fig. 6 shows that the frequency spectra of $V_{\text {upch }}$ and of $i_{\mathrm{sc}}$ have the same structure. Also the cross-correlation function (see fig. 7) underlines the coherence. The formula for the crosscorrelation function is ${ }^{4}$ )

$$
\psi_{12}(\tau)=\frac{1}{2 T} \int_{-T}^{T} f_{1}(t) f_{2}(t+\tau) \mathrm{d} t
$$

in which $\tau$ is the delay time. In the present case $f_{1}(t)$ and $f_{2}(t)$ represent the periodic functions for $V_{\mathrm{upch}}$ and $i_{\mathrm{sc}}$; $T$ is the period of the fundamental frequency, which is the same for both signals. For periodic functions, which can be expanded in Fourier coefficients, one can write:

$$
\psi_{12}(\sigma)=\sum_{n=0}^{\infty} c_{1 n} c_{2 n} \cos \left(n \omega \tau+\phi_{n}\right)
$$

in which $c_{1 n}$ and $c_{2 n}$ represent the amplitudes of the different harmonics and $\phi_{n}$ is a phase angle determined by the phase differences between the corresponding harmonics in the two signals. The cross-correlation function, shown in fig. 7 is built up from the contribution of the different harmonics. Considering the time differences between the points of zero or nearly zero correlation, one can conclude that the best fit to the curve can be obtained with odd harmonics. This is in accordance with the picture developed for the charge transport.

Let us now consider the situation at the belt charge screen. For the upcharge voltage one can write (see fig. 12) $V_{\text {upch }}=E_{\mathrm{b}} d+E_{\mathrm{g}} s+V_{\text {rest }}$, where $E_{\mathrm{b}}$ and $E_{\mathrm{g}}$ are the electric strengths in the belt and in the gas, respectively. The voltage $V_{\text {rest }}$ is the tension between the upcharge screen and the surface of the belt. Because $\varepsilon_{0} \varepsilon_{\mathrm{r}} E_{\mathrm{b}}=D=\sigma$, (with $\varepsilon_{0}=$ the permittivity of vacuum, $\varepsilon_{\mathrm{r}}=$ the relative permittivity, and $D=$ the electric displacement), we can write this formula as

$$
V_{\text {upch }}=\frac{\sigma}{\varepsilon_{0}}\left(\frac{d}{\varepsilon_{\mathrm{r}}}+s\right)+V_{\text {rest }},
$$

with $\sigma=i_{\text {upch }} / b v$. The harmonics in the spectrum of the upcharge voltage (see fig. 5) are caused by fluctuations in the belt velocity, but it is remarkable that the second and fourth harmonics are relatively very strong. For this phenomenon another mechanism is found in the resonances of the belt at the frequencies of the even harmonics as explained in sect. 3.1. Due to the oscillations at the resonance frequencies the belt tension varies accordingly, with the result that $d$ also varies. With a stroboscope it was observed that the moving belt also causes the screen to flap slightly, so $V_{\text {rest }}$ fluctuates. These phenomena all contribute to the strong second and fourth harmonics in the spectrum. The same situation as at the upcharge screen occurs at the collector screen. Because the collector screen is placed in front of the pulley for reasons described in ref. 5 the motion of the screen is less restricted. At the belt resonances, modulation of the voltage over the collector screen, due to the changes in its position, give rise to even harmonics in the spectrum of the collector current.

We would like to thank Prof. A. M. Hoogenboom and Mr R. J. Sparks for valuable discussions.

\section{Appendix}

\section{Vibrations of a moving belt.}

The transverse vibrations which can occur in a belt moving with a definite velocity $v_{0}$ will be analysed here. One considers the simplified model of a moving string, for which the deflections at two fixed points at a distance $l$ are zero. Following the method of d'Alembert one supposes that the possible modes of oscillation of the string can be described by two disturbances moving in opposite directions along the string. On the moving string one takes as a disturbance two sinusoidal waves with the same amplitudes, which propagate in opposite directions. The propagation velocity of the waves 1 and 2 is $v_{1}$ and $v_{2}$, respectively (see fig. 13).

The propagation velocity of transverse waves in a string at rest is given by the well-known formula:

$$
c=\sqrt{ }(S / \rho),
$$

with $S=$ the tension in the string and $\rho=$ the mass per unit length $(1.7 \mathrm{~kg} / \mathrm{m}$ in the present case).

So the velocity of wave 1 is $v_{1}=c+v_{0}$ and of wave $2 v_{2}=c-v_{0}$, referred to a coordinate system at rest. Hence one can write for the amplitudes of the two travelling waves $y_{1}=a \cos \left\{\omega\left(t-x / v_{1}\right)+\phi\right\}$ and

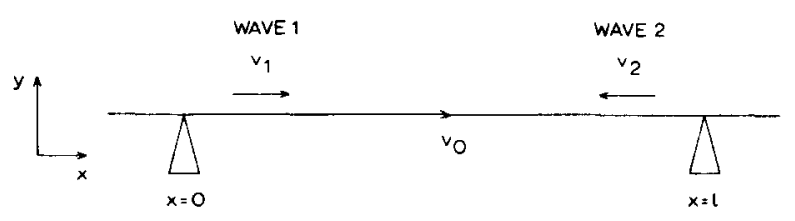

Fig. 13. Model of the belt for the derivation of the belt fluctuations. 
$y_{2}=a \cos \omega\left(t+x / v_{2}\right)$, with $\phi$ an arbitrary phase shift. The two waves interiere at every point of the string. The deflection of a point can then be written as:

$$
\begin{aligned}
y=y_{1}-y_{2}= & 2 a \sin \left\{\omega\left[t-\frac{1}{2} x\left(\frac{1}{v_{1}}-\frac{1}{v_{2}}\right)\right]+\frac{1}{2} \phi\right\} \times \\
& \times \sin \left[\frac{1}{2} \omega x\left(\frac{1}{v_{1}}+\frac{1}{v_{2}}\right)+\frac{1}{2} \phi\right] .
\end{aligned}
$$

The boundary conditions are $y=0$ for $x=0$ and $x=l$, independent of the time $t$ and the phase shift $\phi$. This requires that the second sine term in eq. (3) must be zero, at $x=0$ and $x=l$ for all values of $\phi$. This condition can only be fulfilled if $\phi=0$ and then yields $\frac{1}{2} \omega_{n} l\left(v_{1}^{-1}+v_{2}^{-1}\right)=n \pi, n=0,1,2, \ldots$. One can also write this condition in the form

$$
v_{n}=\frac{n}{2 l} \frac{c^{2}-v^{2}}{c}, \quad n=1,2,3, \ldots,
$$

and one finds then for the deflection of the string

$$
y=2 a \sin \frac{n \pi}{l}\left(\frac{c^{2}-v^{2}}{c}\right)\left(t+\frac{v x}{c^{2}-v^{2}}\right) \sin \frac{n \pi x}{l} .
$$

In the case of a stationary string $(v=0)$ this reduces to the well-known equation for the deflection of a string. For a more rigorous derivation of this formula see refs. 1 and 2.

\section{References}

1) R. Matthieu, VDI Berichte 48 (1961) 71.

2) W. Roth, Antriebstechnik 3 (1964) 48.

3) J. Shaw, private communication (High Voltage Engineering, Burlington, U.S.A.).

4) Y. W. Lee, Statistical theory of communication (J. Wiley, New York, 1960) p. 19.

5) Encyclopedia of Physics 44 (1959) 77. 